\title{
LOS ESTRESORES PSICOSOCIALES SE ASOCIAN A SÍNDROME HIPERTENSIVO DEL EMBARAZO Y/O SÍNTOMAS DE PARTO PREMATURO EN EL EMBARAZO ADOLESCENTE
}

\author{
Maritza Muñoz P. ${ }^{1, a}$, Patricio Oliva M., $P h D^{1, b}$ \\ ${ }^{1}$ Facultad de Ciencias de la Salud y los Alimentos, Universidad del Bío Bío. \\ $a_{\text {Matrona. }}{ }^{b}$ Nutricionista.
}

\section{RESUMEN}

Objetivo: Identificar factores de apoyo y estrés psicosocial en adolescentes embarazadas y establecer la asociación entre estos factores y la presencia o ausencia de síndrome hipertensivo del embarazo (SHE) y/o síntoma de parto prematuro (SPP). Método: Estudio analítico de casos y controles. La muestra estuvo conformada por 146 puérperas adolescentes estratificadas en dos grupos, con y sin SHE y/o SPP durante su gestación. Para describir las variables se realizó un análisis exploratorio de datos utilizando medianas. Para comparar estos valores medios se aplicó la prueba de Mann-Whitney y para el análisis inferencial la prueba $X^{2}$ o Coeficiente V de Cramer. Para determinar el riesgo de presentar el SHE y/o SPP se calculó Odds Ratio. Resultados: Los estresores psicosociales asociados a la presencia de SHE y/o SPP fueron: problemas económicos (OR: 2,46 [1,20 - 5,80]), presencia de agresión psicológica familiar (OR: 4,65 [1,99 - 11,30]), presencia de algún evento estresante y/o depresión (OR: 2,84 [1,36 - 5,98]), presencia de alguna enfermedad y/o hospitalización de un familiar de la adolescente (OR: 6,07 [1,85 - 25,68]), y presencia de violencia al interior del hogar (OR: 4,65 [1,95 - 11,69]). El apoyo familiar se asocia con ausencia de SHE y/o SPP $(p<0,001)$. Conclusiones: Los estresores psicosociales familiares se asocian a la presencia de SHE y/o $\mathrm{SPP}$, mientras que el apoyo familiar es un factor protector de estas patologías.

\section{PALABRAS CLAVE: Embarazo adolescente, síndrome hipertensivo del embarazo, síntomas de par- to prematuro, estrés psicosocial}

\section{SUMMARY}

Objective: To identify support and stress psychosocial factors in pregnant adolescents and to establish association between those factors and the presence or absence of hypertensive syndrome of pregnancy (HSP) and/or symptoms of preterm birth (SPB). Method: Case-control study. The sample consisted of 146 puerperal adolescent women stratified in two groups: with and without HSP and/or SPB during gestation. In order to describe the variables, an exploratory analysis of data was carried out using median. To compare these mean values, Mann-Whitney test was applied. For the analysis of the inferential variables $x^{2}$ test or Cramer's V coefficient was applied. To determine the risk of HSP and/or SPB, the Odds Ratio (OR) was calculated. Results: Psychosocial stressors associated with the presence of HSP and/or SPB are: economic problems (OR: 2.46 [1.20 - 5.80]), presence of family psychological aggression (OR: 4.65 [ 1.99 - 11.30]), presence of a stressful event and/or depression (OR: 2.84 [1.36 - 5.98]), presence of disease and/or hospitalization of a relative of the teenager (OR: 6.07 [1.85 - 25.68]), and presence of violence within the home (OR: 4.65 [1.95 - 11.69]). The family support is associated with the absence of HSP and/or SPB $(p<0.001)$. 
Conclusions: Family psychosocial stressors are mainly associated with the presence of HSP and/or SPB, meanwhile family support is a protective factor for these pathologies.

\section{KEY WORDS: Adolescent pregnancy, hypertensive syndrome of pregnancy, symptoms of preterm birth, psychosocial stress}

\section{INTRODUCCIÓN}

El embarazo adolescente continúa siendo un problema de Salud Pública, a pesar que sus tasas han disminuido desde 1998 a la fecha (1). Los antecedentes disponibles en la bibliografía de los últimos años avalan lo anterior, más aún evidencian una mayor incidencia de patologías del embarazo en adolescentes, encontrándose entre las más frecuentes el síndrome hipertensivo del embarazo (SHE) y el síntoma de parto prematuro (SPP) (26).

Existen numerosos estudios acerca de patologías del embarazo, pero una gran cantidad de ellos han sido realizados desde un enfoque biomédico, no considerándose las exigencias adaptativas de carácter psicosocial que surgen durante la gestación (7), las cuales se ven intensificadas en las adolescentes, que no se encuentran preparadas psicosocialmente para un embarazo, el cual frecuentemente es no deseado (8).

La importancia del entorno psicosocial en el proceso salud-enfermedad se ha documentado en distintos estudios epidemiológicos (7, 9-15), y los planteamientos hipotéticos para explicar esta asociación postulan que la situación psicosocial interviene como un factor condicionante que facilita o inhibe la expresión de factores de riesgo (7). En relación a la importancia del entorno psicosocial durante la gestación, los estresores psicosociales, como estrés y disfunción familiar, han demostrado ser un factor de riesgo con respecto a la morbilidad materna y perinatal, y el apoyo social un factor compensatorio de estos efectos negativos. Por otro lado se ha observado que la evaluación de los factores de riesgo obstétricos conjuntamente con la evaluación de los factores de riesgo psicosociales, incrementan significativamente la probabilidad de identificar a las mujeres embarazadas con mayor riesgo de presentar complicaciones maternas y perinatales (16).

Los antecedentes expuestos y el enfoque de salud familiar vigente en Chile, incentivaron el desarrollo de esta investigación, la cual tuvo por objetivos identificar factores de apoyo y estrés psi- cosocial en adolescentes embarazadas, así como también establecer la asociación entre estos factores psicosociales y la presencia y/o ausencia de SHE y/o SPP en puérperas adolescentes atendidas en los Hospitales Guillermo Grant Benavente de Concepción y Herminda Martín de Chillán, durante el año 2007.

\section{RESULTADOS}

Características generales: De las 146 adolescentes estudiadas el $69,8 \%$ tenía como residencia la ciudad de Concepción y el $30,14 \%$ la ciudad de Chillán. La edad promedio observada fue de $17 \pm$ 1,4 años, con ingresos económicos inferiores a los $\$ 95.000$ (US $\$$ promedio diciembre $2007=\$ 499,28$ ). En su mayoría las adolescentes eran solteras (Tabla I), con actividad de estudiante (Tabla II) y escolaridad media incompleta (Tabla III).

El $99,3 \%$ de las gestantes adolescentes del estudio tuvieron control prenatal durante su gestación (Tabla IV), siendo importante destacar que el $61,6 \%$ $(n=45)$ de los casos presentó SPP, mientras que sólo el 36,9\% ( $n=27)$ presentó SHE, observándose, además, que solamente una de las adolescentes estudiadas presentó ambas patologías (Tabla V).

Tabla I

\section{DISTRIBUCIÓN DE LAS ADOLESCENTES SEGÚN ESTADO CIVIL}

\begin{tabular}{lrrrrrr}
\hline \multirow{2}{*}{ Estado civil } & \multicolumn{2}{c}{ Casos } & \multicolumn{2}{c}{ Controles } & \multicolumn{2}{c}{ Total } \\
& $\mathrm{n}$ & $\%$ & $\mathrm{n}$ & $\%$ & $\mathrm{n}$ & $\%$ \\
\hline Soltera & 42 & 57,5 & 44 & 60,3 & 86 & 58,9 \\
Conviviente & 10 & 13,7 & 11 & 15,1 & 21 & 14,4 \\
Casada & 3 & 4,1 & 1 & 1,4 & 4 & 2,7 \\
Pololeando & 18 & 24,6 & 17 & 23,3 & 35 & 23,9 \\
Total & 73 & 100,0 & 73 & 100,0 & 146 & 100,0 \\
\hline
\end{tabular}

Cramer's V=0,0877 (NS) 
Tabla II

\section{DISTRIBUCIÓN DE LAS ADOLESCENTES SEGÚN ACTIVIDAD}

\begin{tabular}{lrrrrrrr}
\hline Actividad & \multicolumn{2}{c}{ Casos } & \multicolumn{2}{c}{ Controles } & \multicolumn{2}{c}{ Total } \\
& $\mathrm{n}$ & $\%$ & $\mathrm{n}$ & $\%$ & $\mathrm{n}$ & $\%$ \\
\hline Estudiante & 34 & 46,6 & 39 & 53,4 & 73 & 50,1 \\
Ninguna & 15 & 20,6 & 16 & 21,9 & 31 & 21,2 \\
Dueña de casa & 24 & 32,9 & 11 & 15,1 & 35 & 23,9 \\
Trabaja & 0 & - & 7 & 9,6 & 7 & 4,8 \\
Total & 73 & 100,0 & 73 & 100,0 & 146 & 100,0
\end{tabular}

Cramer's V=0,2891 (NS)

Tabla III

DISTRIBUCIÓN DE LAS ADOLESCENTES SEGÚN ESCOLARIDAD

\begin{tabular}{lccccccc}
\hline \multirow{2}{*}{ Escolaridad } & \multicolumn{2}{c}{ Casos } & \multicolumn{2}{c}{ Controles } & \multicolumn{2}{c}{ Total } \\
& $\mathrm{n}$ & $\%$ & $\mathrm{n}$ & $\%$ & $\mathrm{n}$ & $\%$ \\
\hline Sin estudios & 1 & 1,4 & 2 & 2,7 & 3 & 2,1 \\
Básica incompleta & 15 & 20,6 & 3 & 4,1 & 18 & 12,3 \\
Básica completa & 4 & 5,5 & 2 & 2,7 & 6 & 4,1 \\
Media incompleta & 43 & 58,9 & 45 & 61,6 & 88 & 60,3 \\
Media completa & 10 & 13,7 & 21 & 28,8 & 31 & 21,2 \\
Total & 73 & 100,0 & 73 & 100,0 & 146 & 100,0 \\
\hline
\end{tabular}

Cramer's V=0,2978 (NS)

Tabla IV

DISTRIBUCIÓN DE LAS ADOLESCENTES SEGÚN NÚMERO DE CONTROLES PRENATALES

\begin{tabular}{lcccccc}
\hline & \multicolumn{2}{c}{ Casos } & \multicolumn{2}{c}{ Controles } & \multicolumn{2}{c}{ Total } \\
Control prenatal & $\mathrm{n}$ & $\%$ & $\mathrm{n}$ & $\%$ & $\mathrm{n}$ & $\%$ \\
\hline No tuvo controles & 0 & - & 1 & 1,4 & 1 & 0,7 \\
$\leq 3$ & 0 & - & 3 & 4,1 & 3 & 2,1 \\
4 a 5 & 7 & 9,6 & 7 & 9,6 & 14 & 9,6 \\
$>5$ & 66 & 90,4 & 62 & 84,9 & 128 & 87,6 \\
Total & 73 & 100,0 & 73 & 100,0 & 146 & 100,0 \\
\hline
\end{tabular}

Tabla V

\section{DISTRIBUCIÓN DE LAS ADOLESCENTES SEGÚN PATOLOGÍA DEL EMBARAZO}

\begin{tabular}{lccccccc}
\hline \multirow{2}{*}{ Patologías } & \multicolumn{2}{c}{ Casos } & \multicolumn{2}{c}{ Controles } & \multicolumn{2}{c}{ Total } \\
& $\mathrm{n}$ & $\%$ & $\mathrm{n}$ & $\%$ & $\mathrm{n}$ & $\%$ \\
\hline SPP & 42 & 57,5 & 0 & - & 42 & 28,8 \\
SHE & 26 & 35,6 & 0 & - & 26 & 17,8 \\
Otra patología & 0 & - & 8 & 10,9 & 8 & 5,5 \\
Ninguna patología & 0 & - & 65 & 98,1 & 65 & 44,5 \\
SPP más SHE & 1 & 1,4 & 0 & - & 1 & 0,7 \\
Otra más SPP & 3 & 4,1 & 0 & - & 3 & 2,1 \\
Otra más SHE & 1 & 1,4 & 0 & - & 1 & 0,7 \\
Total & 73 & 100,0 & 73 & 100,0 & 146 & 100,0
\end{tabular}

Cramer's $\mathrm{V}=1,0$

Factores de apoyo psicosocial: El grupo control presentó mayor apoyo familiar $(91,4 \%)$, de la pareja $(75,3 \%)$ y de la comunidad escolar $(58,9 \%)$ durante su embarazo, que el grupo de los casos $(67,12 \%$, $65,75 \%$ y 35,62 respectivamente). Solo se observó lo contrario en el apoyo de los amigos, en donde los casos presentaron un $93,5 \%$ y los controles un $84,9 \%$.

Factores de estrés psicosocial: En el grupo de casos los estresores psicosociales de mayor frecuencia fueron la percepción de problemas económicos $(56,1 \%)$, percepción de insuficiencia de dinero disponible $(53,4 \%)$, presencia de eventos estresantes y/o depresión $(53,4 \%)$, presencia de agresión psicológica familiar $(45,2 \%)$ y presencia de violencia psicológica, física o sexual al interior del hogar $(42,4 \%)$, valores muy superiores a los encontrados en el grupo de controles $(34,2 \%, 28,7 \%$, $15,1 \%$ y $13,7 \%$ respectivamente), con la sola excepción de la percepción de insuficiencia de dinero disponible, en que se observó igualdad en ambos grupos $(53,4 \%)$.

Factores psicosociales asociados: La presencia de estresores psicosociales se asoció significativamente a un mayor riesgo de presentar SHE y/o SPP en el embarazo adolescente. Estos fueron: problemas económicos, agresión psicológica familiar, algún evento estresante y/o depresión, enfermedad y/o hospitalización de un familiar, y violencia familiar (Tabla VI). El apoyo familiar se asocia significativamente $(78 \%)$ con la ausencia de SHE y/o SPP $(p<0,001)$, por lo que constituye un factor protector de esas patologías. 


\section{Tabla VI}

RIESGO DE SÍNDROME HIPERTENSIVO DEL EMBARAZO Y/O PARTO PREMATURO SEGÚN PRESENCIA DE ESTRESORES PSICOSOCIALES

\begin{tabular}{|c|c|c|}
\hline Estresores psicosociales & OR & IC95\% \\
\hline Problemas económicos & 2,46 & $1,2-5,80$ \\
\hline Agresión psicológica familiar & 4,65 & $1,99-11,30$ \\
\hline $\begin{array}{l}\text { Algún evento estresante } \\
\text { y/o depresión }\end{array}$ & 2,84 & $1,36-5,98$ \\
\hline $\begin{array}{l}\text { Enfermedad y/o hospitalización } \\
\text { de un familiar }\end{array}$ & 6,07 & $1,85-25,68$ \\
\hline Violencia al interior del hogar & 4,65 & $1,95-11,69$ \\
\hline
\end{tabular}

\section{DISCUSIÓN}

Nuestro estudio confirma la asociación entre la presencia de estresores psicosociales y el mayor riesgo de presentar síndrome hipertensivo del embarazo y/o parto prematuro, en el embarazo adolescente.

Autores de diversos estudios realizados en embarazadas adolescentes, han reportado que una de las complicaciones obstétricas más frecuentes es el desarrollo del SHE, publicando valores de frecuencias entre $22,4 \%$ y $29 \%(3,17)$, cifras que son levemente superiores a la encontrada en nuestro estudio, en el cual fue posible observar que la frecuencia alcanzó un 19,2\% de las adolescentes embarazadas. En cambio en lo que respecta al SPP, Calderón y cols (18), reportaron una incidencia de 5 a $10 \%$ del total de nacimientos, porcentaje muy por debajo a la frecuencia de $31,5 \%$ encontrada en esta investigación.

Un reciente estudio realizado por Leeners y cols (19), señalaron que el estrés emocional durante el embarazo representaba un efecto significativo en la incidencia de desordenes hipertensivos del embarazo. Otras investigaciones han demostrado, que la exposición a factores estresantes crónicos inducen a alteraciones a nivel de la microcirculación úteroplacentaria y función placentaria que desencadenarían patologías como el SHE (20). Al mismo tiempo se ha reportado cada vez mayor evidencia que sustenta la asociación entre un número de factores de riesgo modificables y $\operatorname{SPP}(21,22)$. Diversos autores sostienen que las tensiones psicosociales como la violencia durante el embarazo y el estrés, pueden desencadenar un trabajo de parto prematu- ro, con la participación de algunas sustancias iberadas durante una situación de estrés, tales como las prostaglandinas $(16,18,21,23)$. Estos hallazgos son concordantes con lo encontrado en el presente estudio, donde fue posible constatar que la agresión psicológica familiar y la presencia de algún evento estresante y/o depresión durante la gestación de la adolescente están asociados significativamente $(p<0,001, p<0,005$ respectivamente) a la presencia de SPP y/o SHE.

Alvarado y cols (24), observaron que uno de los eventos vitales que con mayor frecuencia se presentó en el embarazo fue la enfermedad de un familiar $(16,7 \%)$, cifra similar a la encontrada en la presente investigación (15,7\%). Asimismo se pudo observar que esta variable estaba asociada como factor de riesgo a SHE y/o SPP en las adolescentes estudiadas.

Por otro lado, al analizar la variable violencia en el hogar, fue posible constatar que existió una asociación significativa con la presencia de SHE y/o SPP $(p<0,001)$, hallazgos que podrían sustentar y/o confirmar que la violencia doméstica, constituye un factor psicosocial subyacente condicionante de respuestas maternas adversas en el embarazo, siendo el SHE y SPP dos de sus expresiones físicas de mayor trascendencia biomédica $(20,25)$.

La disfunción familiar ha demostrado ser un factor de riesgo con respecto a la morbilidad materna y perinatal (16). En una investigación publicada por Wang y cols (15), se señala que el estrés producido a nivel familiar puede acelerar el proceso de enfermedades coronarias en mujeres. Esto podría concordar con lo reportado en los resultados del presente estudio, en el cual se observó que el apoyo familiar durante el embarazo se asoció significativamente a la ausencia de SHE y/o SPP $(p<0,001)$, en donde las adolescentes que no presentaban las patologías y al mismo tiempo señalaron que recibieron apoyo familiar durante el período de gestación correspondieron al $90,4 \%$ de la muestra.

\section{CONCLUSIÓN}

Los estresores psicosociales familiares como enfermos en la familia, violencia intrafamiliar, agresión psicológica familiar y eventos estresantes, se asocian a la presencia de SHE y/o SPP en gestantes adolescentes, siendo el apoyo familiar, un factor protector de esas patologías.

\section{BIBLIOGRAFÍA}

1. Ministerio de Salud (2007), Manual de Atención Personalizada con Enfoque Familiar en el Proceso 
Reproductivo, Departamento del Ciclo Vital. Hallado en: http://www.redsalud.gov.cl/archivos/ciclovital/saludreproductiva.pdf

2. Guerra CL, Vásquez A, Herrera $V$, de la Cruz F, Almirall AM. Embarazo y adolescencia: Factores biológicos materno y perinatal más frecuentes. Rev Cubana Obstet Ginecol 2001;27(2):165-71.

3. Díaz A, Sanhueza P, Yaksic N. Riesgos obstétricos en el embarazo adolescente: estudio comparativo de resultados obstétricos y perinatales con pacientes embarazadas adultas. Rev Chil Obstet Ginecol 2002;67(6):481-7.

4. Gigante DP, Victora CG, Gonçalves H, Lima RC, Barros FC, Rasmussen KM. Risk factors for childbearing during adolescence in a population-based birth cohort in southern Brazil. Pan Am J Public Health 2004;16(1):1-10.

5. Sibai B, Dekker G, Kupferminc M. Pre-Eclampsia. Lancet 2005;365(9461):785-99.

6. Dissanayake VH, Samarasinghe HD, Morgan L, Jayasekara RW, Seneviratne HR, Broughton F. Morbidity and mortality associated with pre-eclampsia at two tertiary care hospitals in Sri Lanka. J Obstet Gynaecol 2007;33(1):56-62.

7. Salvador-Moysén J, Martínez-López Y, LechugaQuiñones A, Ruiz-Astorga R, Terrones-González A. Situación psicosocial de las adolescentes y toxemias del embarazo. Rev Salud Púb México 2000;42(2):99105.

8. Mirabal G, Martínez MM, Pérez D. Repercusión biológica, psíquica y social del embarazo en la adolescencia. Rev Cubana Enferm 2002;18(3):175-83.

9. Rozanski A, Blumenthal JA, Kaplan J. Impact of psychological factors in the pathogenesis of cardiovascular disease and implications for therapy. Circulation 1999;99(16):2192-217.

10. Antoni MH, Lutgendorf S. Psychosocial factors and disease progression in cancer. Curr Dir Psychol Sci 2007;16(1):42-46.

11. De Backer G, Ambrosioni E, Borch-Johnsen K, Brotons C, Cifkova R, Dallongeville J, et al. European guidelines on cardiovascular disease prevention in clinical practice. Third Joint Task Force of European and other Societies on Cardiovascular disease prevention in clinical practice. Arch Mal Coeur Vaiss 2004;97(10):1019-30.

12. Yusuf S, Hawken S, Ôunpuu S, Dans T, Avezum A, Lanas $F$, et al. Effect of potentially modifiable risk factors associated with myocardial infarction in 52 countries (The Inter Heart Study): case-control study. Lancet 2004;364(9438):937-52.

13. Dolic M, Bailer J, Staehle HJ, Eickholz P. Psychoso- cial factors as risk indicators of periodontitis, $\mathrm{J}$ Clin Periodontol 2005;32(11):1134-40.

14. Lucini D, Di Fede G, Parati G, Pagani M. Impact of chronic psychosocial stress on autonomic cardiovascular regulation in otherwise healthy subjects. Hypertension 2005;46(5):1201-6.

15. Wang HX, Leineweber C, Kirkeeide R, Svane B, Schenck-Gustafsson K, Theorell T, et al. Psychosocial stress and atherosclerosis: family and work stress accelerate progression of coronary disease in women. The Stockholm Females Coronary Angiography Study. J Internal Med 2007;261(3):245-54.

16. Herrera JA, Ersheng G, Shahabuddin AK, Lixia D, Wei $Y$, Faisal M, et al. Evaluación periódica del riego biopsicosocial prenatal en la predicción de las complicaciones maternas y perinatales en Asia 2002-2003. Colombia Médica 2006;37(2-1):6-14.

17. Ciero M, Rodríguez D, Fernández-Llimos F. Hipertensión Arterial: riesgos para la madre y el bebé, Seguim Farmacoter 2003;1(3): 91-98.

18. Calderón J, Vega G, Velásquez J, Morales R, Vega AJ. Factores de riesgo materno asociados al parto pretérmino. Rev Méd IMSS 2005;43(4):339-42.

19. Leeners B, Neumaier-Wagner P, Kuse S, Stiller R, Rath W. Emotional stress and the risk to develop hypertensive diseases in pregnancy, Hypertens Pregnancy 2007;26(2):211-26.

20. Arcos E, Uarac M, Molina I, Repossi A, Ulloa M. Impacto de la violencia doméstica sobre la salud reproductiva y neonatal. Rev Méd Chil 2001;129(12):141324.

21. Heaman MI, Blanchard JF, Gupton AL, Moffat ME, Currie RF. Risk factors for spontaneous preterm birth among aboriginal and non-aboriginal women in Manitoba. Paediatr Perinat Epidemiol 2005;19(3):181-93.

22. Beth L. Psychosocial pathways to prematurity: changings our thinking toward a lifecourse and community approach. J Obstet Gynecol Neonatal Nurs 2003;32(5):650-8.

23. Mulder EJ, Robles de Medina PG, Huizink AC, Van den Bergh BR, Buitelaar JK, Visser GH. Prenatal maternal stress: effects on pregnancy and the (unborn) child. Early Hum Dev 2002;70(1-2):3-14.

24. Alvarado R, Medina E, Aranda W. El efecto de variables psicosociales durante el embarazo, en el peso y la edad gestacional del recién nacido, Rev Méd Chil 2002;130(5):561-8.

25. Covington DL, Justason BJ, Wright LN. Severity, manifestations, and consequences of violence among pregnant adolescents. J Adolescent Health 2001;28(1):55-61. 